\section{FRI0645 NAILFOLD CAPILLAROSCOPY IN DIABETES MELLITUS TYPE 2}

G. Maldonado $^{1}$, C. Paredes ${ }^{2}$, R. Guerrero ${ }^{1}$, C. Ríos ${ }^{1} .{ }^{1}$ Universidad Espíritu Santo; ${ }^{2}$ Universidad Espiritu Santo, Guayaquil, Ecuador

Background: Diabetes mellitus (DM) is characterized by chronic hyperglycemia states and the development of specific microvascular disorders such as retinopathy and nephropathy. Conventional methods are usually used to study the vascular compromise of this entity, however, the use of capillaroscopy for the evaluation of capillary microarchitecture is not frequently used.

Objectives: The objective of this study was to identify vascular alterations in patients with type II diabetes mellitus and to determine the relationship between capillaroscopic findings and clinical manifestations.

Methods: Observational, descriptive and prospective of patients with diabetes mellitus II selected by inclusion and exclusion criteria. Subjects presenting signs/symptoms or history of any collagen disease, trauma presence in the nailfold due to cosmetic treatment or nail polish, were excluded. The capillaroscopy was performed by an experienced rheumatologist in a room with an ambient temperature of $20-230 \mathrm{C}$. The fourth and fifth fingers of the nondominant hand were chosen. The capillaries were observed using a 10x magnification capillaroscope (Dino-Lite) and photographs of the last distal row of capillaries were taken. The following capillaroscopic parameters were considered: capillary diameter (ectasia and giant capillaries), crosslinked capillaries, capillary tortuosity, ramified capillaries, avascular zones, hemorrhages, dominant morphology, subpapillar venous plexus visibility, cuticulitis and SD pattern. The images were analyzed by an experienced rheumatologist. Data was analyzed using SPSS. The nonparametric correlations were performed by tau $b$ Kendall and values were considered statistically significant when $p>0.01$ and they had two tails.

Results: 65 patients were included in the study, with a mean age of 57 years [39-80], of which $75 \%$ [49] were women and $25 \%$ [16] men. The capillaroscopic findings were evident in $83 \%$ of the study population. The most frequent alterations were tortuous capillaries in 63\% [41], cross-linked capillary in 59\% [38], avascular areas in $48 \%$ [34], ectasias in 31\% [25]. In smaller frequency, giant capillaries $14 \%$ [9], arborified capillaries $11 \%$ [7], no haemorrhages, no SD pattern. The capillaroscopic findings representing vascular damage were greater in patients with Diabetes Mellitus than in the control group (Figure 1). Moreover, the capillary morphology in the control group was open versus tortuous in patients with DM. Those patients with capillaroscopic alterations had a longer time of evolution of the disease with an average of 12.8 years, compared to those who did not present alterations that had a mean evolution of the disease of 8.5 years, which shows that those capillaroscopic alterations represent progressive endothelial damage. In addition, an association between the presence of retinopathy and capillary damage at the nail bed level was demonstrated $(p>0.001)$.

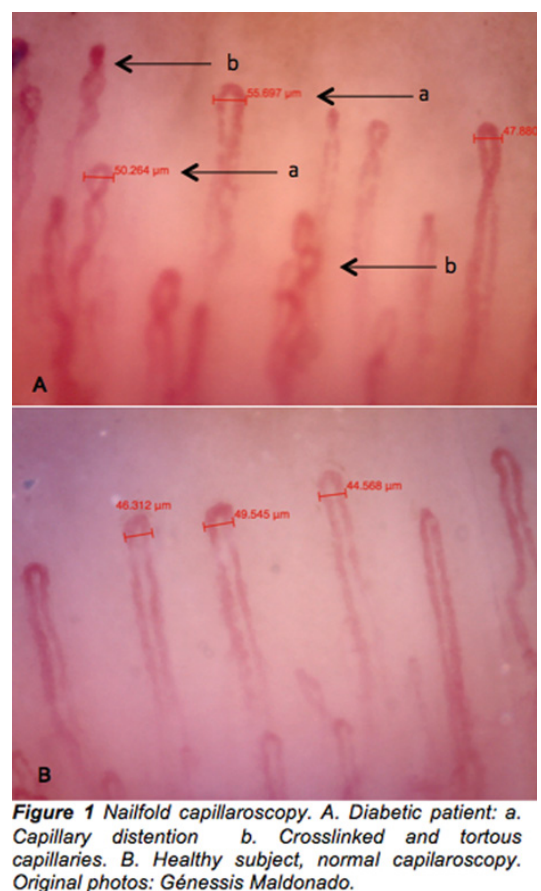

Conclusions: Capillaroscopy has proven to be a non-invasive, reproducible and reliable technique for the evaluation of vascular microarchitecture within a large group of rheumatic diseases of the scleroderma spectrum. However, it has been shown that it can be used for evaluation other diseases outside the field of Rheumatology such as diabetes, being a tool that should be known by primary care physicians and healthcare workers.

Disclosure of Interest: None declared

DOI: 10.1136/annrheumdis-2017-eular.1422

\section{FRI0646 THREE-DIMENSIONAL NAIL IMAGING BY OPTICAL COHERENCE TOMOGRAPHY: A NOVEL BIOMARKER OF RESPONSE TO THERAPY FOR NAIL DISEASE IN PSORIASIS AND PSORIATIC ARTHRITIS}

G. Abignano ${ }^{1,2}$, P. Laws ${ }^{3}$, F. Del Galdo ${ }^{1,2}$, H. Marzo-Ortega ${ }^{1,2}$,

D. McGonagle ${ }^{1,2} .{ }^{1}$ Leeds Institute of Rheumatic and Musculoskeletal Medicine, University of Leeds; ${ }^{2}$ NIHR Leeds Musculoskeletal Biomedical Research Unit, Leeds Teaching Hospitals NHS Trust; ${ }^{3}$ Department of Dermatology, Leeds Teaching Hospitals NHS Trust, Leeds, United Kingdom

Background: Nail disease is a common feature of psoriasis and psoriatic arthritis (PsA) and can impact quality of life significantly. The assessment of nail disease, unlike skin disease, is challenging with no tissue biomarkers feasible. Commonly used clinical outcomes include the Nail Psoriasis Severity Index (NAPSI) or the modified NAPSI system which are subjective. Optical Coherence Tomography (OCT) can detect changes in psoriatic nails and is a potential tool to assess response to therapy (1).

Objectives: To evaluate OCT imaging changes in cases with nail psoriasis following 6 month-therapy with Apremilast, a PDE4 inhibitor with known efficacy for nail disease in psoriasis and PsA.

Methods: Forty fingernails from four psoriatic patients were imaged at baseline and after 6 month-treatment with Apremilast $30 \mathrm{mg}$ bd using Vivosight OCT scanner (Michelson Diagnostics Ltd., Kent, UK). Three OCT scans were collected from each fingernail ( 1 transverse and 2 longitudinal - proximal and distal) totalling 240 scans available for analysis. OCT scoring of the forty fingernails, at baseline and six months later, was carried out for changes including: 1. leukonychia/white spots; 2. pitting/localized surface irregularities; 3 . diffuse surface waving; 4. onycholysis; 5 . subungual hyperkeratosis. OCT score was arbitrarily calculated based on the absence $(=0)$ or presence $(=1)$ of each feature on the three scans of the corresponding fingernail (range $0-15$ ). Macroscopic nail features were scored at baseline and 6 months later using the NAPSI scoring system by a Dermatologist blinded to OCT findings and compared with OCT score. Data were expressed as median (range). Comparison between baseline and follow up measurements was performed using Wilcoxon matched-pairs signed rank test. Difference between two groups and correlation were calculated using Mann-Whitney test and Spearman's test respectively. Statistical analysis was carried out using GraphPad Prism software V.7.0.

Results: Based on NAPSI, twenty-eight/40 fingernails (70\%) improved or remained stable after 6 months with the median NAPSI per nail falling from 3.5 $(0-7)$ to $0(0-6)(p<0.0001)$. The OCT evaluation showed that the entire gamut of nail changes exhibited improvement or stabilization in thirty-three/40 $(82.5 \%)$ nails after 6 months with median OCT score per nail falling from $8(3-14)$ to 4 $(1-9)(p<0.0001)$. Difference of the change $(\Delta)$ of OCT score between baseline and follow-up significantly correlated with $\Delta$-NAPSI $(r=0.71, p<0.0001)$.

Conclusions: OCT is able to identify all common psoriasis related nail pathology and changes after treatment. Further studies with larger numbers are needed to validate its potential role as a biomarker for nail disease.

References:

[1] Aydin SZ et al. Dermatology 2013.

Disclosure of Interest: None declared

DOI: 10.1136/annrheumdis-2017-eular.4157

\section{FRI0647 COLOR DOPPLER AS A VALUABLE PROCEDURE FOR EARLY DIAGNOSIS OF OCULAR VASCULAR CHANGES IN BEHÇET'S DISEASE}

H.H. Al Sherbeni ${ }^{1}$, S. Metawie ${ }^{1}$, R. Abdel salam ${ }^{2}$, H. Azizi ${ }^{3} \cdot{ }^{1}$ Rheumatology and Rehabilitation; ${ }^{2}$ Ophthalmology; ${ }^{3}$ Radiology, Faculty of Medicine- Cairo University, Cairo, Egypt

Background: Ocular involvement is a common and serious manifestation of Behçet's disease (BD). It occurs in about $70 \%$ of the patients and is associated with a high risk of blindness (Kaçmaz et al., 2008).

Color Doppler is used for imaging retrobulbar vasculature. It measures the peak systolic velocity (PSV) and end diastolic velocity (EDV), from which the resistance index $(R I)$ is calculated in the ophthalmic artery (OA), central retinal artery (CRA), and posterior ciliary artery (PCA) to detect any abnormality in the retrobulbar vessels (Nagaoka, 2006).

Objectives: To determine and compare the orbital Color Doppler haemodynamic parameters of Behçet's patients with those of healthy subjects and to evaluate the OA, CRA, PCA flow velocities and resistance indices (RIs).

Methods: This study included thirty patients suffering from Behçet's disease satisfying at least 3 of the criteria of BD according to the International Study Group for Behçet's disease in 1990. 20 age/sex-matched healthy subjects served as a control group. All patients were subjected to history taking, clinical examination, full ophthalmological examination, routine laboratory investigations and Color Doppler ultrasound (CDU) examination.

Results: The patients' age ranged from 18 to 45 years with a mean of $31.0 \pm 7.5$. Their disease duration ranged from 0.25 to 20 years with a mean of $5.71 \pm 5.84$. There were diminished blood flow velocities of the OA, CRA and PCA as the peak systolic velocity (PSV) and end diastolic velocity (EDV) of these arteries were lower than normal while resistance flow indices (RIs) were higher than normal. 
Table 1. Results of ocular Doppler ultrasound over right (RT) and left (LT) sided arteries

\begin{tabular}{|c|c|c|c|c|c|c|c|c|c|c|}
\hline & \multicolumn{5}{|c|}{ RT sided arteries } & \multicolumn{5}{|c|}{ LT sided arteries } \\
\hline & & Range & Mean & S.D & P-value & & Range & Mean & S.D & P-value \\
\hline \multirow[t]{2}{*}{ OA-PSV } & $P$ & $6.7-57$ & 27.5 & 13.5 & 0.001 & $P$ & $7.7-65$ & 26.37 & 12.26 & 0.000 \\
\hline & C & $34-40$ & 36.9 & 2.04 & & r & $32-43.8$ & 8.73 & 3.17 & \\
\hline \multirow[t]{2}{*}{ OA-EDV } & $P$ & $0.0-16.3$ & 6.9 & 4.06 & 0.000 & $P$ & $0.4-22.4$ & 6.69 & 4.17 & 0.002 \\
\hline & C & $9-12.3$ & 10.6 & 1.33 & & C & $7.6-12.3$ & 10.36 & 1.52 & \\
\hline \multirow[t]{2}{*}{ OA-RI } & $P$ & $0.63-1$ & 0.76 & 0.08 & 0.020 & $\mathrm{P}$ & $0.62-0.95$ & 0.75 & 0.08 & 0.123 \\
\hline & C & $0.67-0.79$ & 0.72 & 0.72 & & C & $0.67-0.78$ & 0.73 & 0.03 & \\
\hline \multirow[t]{2}{*}{ CRA-PSV } & $P$ & $6.5-37.4$ & 13.2023 & 6.53 & 0.288 & $\mathrm{P}$ & $4.3-37.1$ & 12.61 & 7.68 & 0.949 \\
\hline & C & $9.4-$ & 11.84 & 1.52 & & C & 8.5 & 12.51 & 1.91 & \\
\hline \multirow[t]{2}{*}{ CRA-EDV } & $\mathrm{P}$ & 1.6 & & 2.13 & 0.200 & $\mathrm{P}$ & 1.6 & 4.84 & 4.95 & 0.466 \\
\hline & C & 2. & & 3.57 & & $\mathrm{C}$ & 2.6 & 4.13 & 0.913 & \\
\hline \multirow[t]{2}{*}{ CRA-RI } & $P$ & 0.45 & 0. & 0.07 & 0.917 & $\mathrm{P}$ & $0.27-0.80$ & 0.66 & 0.096 & 0.883 \\
\hline & C & 0.61 & 0.6 & 0.03 & & C & $0.61-0.71$ & 0.67 & 0.037 & \\
\hline \multirow[t]{2}{*}{ PCA-PSV } & $P$ & $6-45.7$ & 13.82 & 8.35 & 0.352 & $\mathrm{P}$ & $6.1-21.6$ & 13.4 & 4.22 & 0.872 \\
\hline & C & $9.9-17.3$ & 13.53 & 2.02 & & C & $10-15.4$ & 13.5 & 1.57 & \\
\hline \multirow{2}{*}{ PCA-EDV } & $P$ & $2.2-11.4$ & 4.96 & 2.67 & 0.408 & $\mathrm{P}$ & $1.9-7.32$ & 4.7 & 1.65 & 0.386 \\
\hline & C & $3.5-6.1$ & 5.16 & 0.99 & & C & $3.2-6.1$ & 5.04 & 0.96 & \\
\hline \multirow[t]{2}{*}{ PCA-RI } & $P$ & $0.49-0.76$ & 0.63 & 0.06 & 0.409 & $P$ & $0.53-0.68$ & 0.87 & 1.16 & 0.435 \\
\hline & C & $0.6-0.68$ & 0.63 & 0.02 & & C & $0.59-0.69$ & 0.63 & 0.029 & \\
\hline
\end{tabular}

$\mathrm{C}=$ Control, $\mathrm{LT}=$ Left, $\mathrm{P}=$ Patients, $\mathrm{RT}=$ Right, $\mathrm{S} . \mathrm{D}=$ Standard Deviation.

Conclusions: Behçet's disease patients with ocular involvement have lower CRA, PCA and OA blood flow velocities than healthy control. CDU is helpful in early diagnosis of ocular Behçet's disease activity as it is a widely used, easy-to-perform and accurate method.

\section{References:}

[1] Kaçmaz O., Kempen J.H., Newcomb C., Gangaputra S., Daniel E., et al.(2008): The Systemic Immunosuppressive Therapy for Eye Diseases: Cohort Study Group. Am J Ophthalmol; 146(6): 828-836.

[2] Nagaoka T. (2006): Physiological mechanism for the regulation of ocular circulation: Nippon Ganka Gakkai Zasshi. 110 (11): 872-8.

[3] The International Study Group for Behçet's disease (1990): Criteria for diagnosis of Behçet's disease. Lancet; 335:1078-1080.

Disclosure of Interest: None declared

DOI: 10.1136/annrheumdis-2017-eular.4113

\section{FRI0648 CONCORDANCE BETWEEN THE TUBERCULIN SKIN TEST AND INTERFERON GAMMA RELEASE ASSAY FOR DIAGNOSING LATENT TUBERCULOSIS INFECTION IN PATIENTS WITH CHRONIC INFLAMMATORY ARTHRITIS}

I. Aktas $^{1}$, F. Unlu Ozkan ${ }^{1}$, T. Nazligul ${ }^{1}$, H. Kuzu Okur ${ }^{2} .{ }^{1}$ Department of Physical Medicine and Rehabilitation; ${ }^{2}$ Department of Chest Disease, Fatih Sultan Mehmet Education and Research Hospital, Istanbul, Turkey

Background: Biologic therapies have led to a dramatic change in the management of rheumatologic diseases. Although biologic agents provide profound clinical benefits, various adverse events are associated with their use particularly an increased risk of the reactivation of latent tuberculosis. Therefore, screening for latent tuberculosis infection (LTBI) is imperative before initiating biologic agents ${ }^{1}$. Although tuberculin skin test (TST) is the most common test for detecting LTBI, Bacillus Calmette-Gue'rin (BCG) vaccination and non-tuberculosis Mycobacterium infections, can lead to false positive TST results. Interferon-gamma release assay (IGRA) measures the immune response to tuberculosis specific antigens that do not cross react with BCG, and therefore, do not cause false positive reactions in $B C G$ recipients. Its positiveness indicates the presence of tuberculosis infection either latent or active ${ }^{2}$.

Objectives: We aimed to prevent the unnecessary anti-tuberculosis prophylaxis in patients whom biologic therapy is planned by evaluating the concordance between the TST and IGRA.

Methods: Patients who have been receiving biologic therapy due to chronic inflammatory arthritis were enrolled in this study. Demographic and clinical data, TST and IGRA results were recorded. The agreement between IGRA and TST results was evaluated by Kappa coefficient.

Results: A total of 35 patients were included; 15 (42.8\%) were male and mean age was $43.74 \pm 12.72$ years. Of the 22 TST positive patients, $13(37.1 \%)$ were IGRA negative. Of the 13 TST negative patients, $3(8.6 \%)$ were IGRA positive. Nine $(25.7 \%)$ patients were positive for either of the two tests and 10 $(28.6 \%)$ patients were negative for both tests. There was statistically significant discordance between two tests $(p: 0.021 ; p<0.05)$ (Table 1$)$. While positive rate of TST was $62.9 \%$, positive rate of IGRA was $34.3 \%$ and Kappa consistency coefficient between two tests was $15.4 \%$ ( $p: 0.283 ; p>0.05)$.

Table 1. Agreement between IGRA and TST results

\begin{tabular}{lcccc}
\hline IGRA & \multicolumn{2}{c}{ TST } & Total & $\mathrm{p}$ \\
\cline { 2 - 3 } & Negative $\mathrm{n}(\%)$ & Positive $\mathrm{n}(\%)$ & $\mathrm{n}(\%)$ & \\
\hline Negative & $10(28.6)$ & $13(37.1)$ & $23(65.7)$ & $\mathbf{0 . 0 2 1}$ \\
Positive & $3(8.6)$ & $9(25.7)$ & $12(34.3)$ & \\
Total & $13(37.1)$ & $22(62.9)$ & & \\
\hline
\end{tabular}

Conclusions: It is very common in rheumatology practice to administer antituberculosis prophylaxis according to the TST. IGRA may reduce the number of patients in whom tuberculostatics are prescribed, especially in BCG recipients in endemic populations, resulting in a benefit of avoiding possible side effects. Furthermore, IGRA is also important for detecting the cases of LTBI that would be missed by TST. Confirmation in larger studies is necessary.

References:

[1] Costantino F, de Carvalho Bittencourt M, Rat AC, et al. Screening for latent tuberculosis infection in patients with chronic inflammatory arthritis: discrepancies between tuberculin skin test and interferon- $\gamma$ release assay results. J Rheumatol. 2013 Dec;40(12):1986-93.

[2] Ruan Q, Zhang S, Ai J, et al. Screening of latent tuberculosis infection by interferon- $\gamma$ release assays in rheumatic patients: a systemic review and meta-analysis. Clin Rheumatol. 2016 Feb;35(2):417-25.

Disclosure of Interest: None declared

DOI: 10.1136/annrheumdis-2017-eular.5712

\section{FRI0649 STANDARDIZED PROCEDURES FOR ULTRASOUND IMAGING IN PAEDIATRIC RHEUMATOLOGY: PROGRESS OF A EULAR TASK FORCE}

I. Janta ${ }^{1}$, P. Collado Ramos ${ }^{2}$, J. Vojinovic ${ }^{3}$, E. Naredo ${ }^{4}$, L. Carmona ${ }^{5}$.

${ }^{1}$ Rheumatology, Hospital General Universitario Gregorio Marañón;

${ }^{2}$ Rheumatology, Rheum-Pediatric Unit, Hospital Universitario Severo Ochoa,

Madrid, Spain; ${ }^{3}$ Pediatric Rheumatology, Clinical Center, Faculty of Medicine,

University of Nis, Nis, Serbia; ${ }^{4}$ Rheumatology, Joint and Bone Research Unit,

Hospital Universitario Fundación Jiménez Díaz and Autónoma University;

${ }^{5}$ Instituto de Salud Musculoesquelética, Madrid, Spain

Background: Musculoskeletal ultrasound (MSUS) is a useful imaging technique in paediatric rheumatic diseases (PRD). US has several advantages over other imaging techniques: it is non-invasive, radiation free, rapid, highly accepted by the patients and does not require sedation for scanning in younger children. However, MSUS examination is incriminated to be an operator dependent technique. Moreover, the variability of normal sonoanatomy in children, due to ossification, makes the acquisition and interpretation of MSUS images difficult. The variability in background and experience of ultrasonographers in different countries requires an international multidisciplinary effort for an optimal standardization of MSUS performance in PRD.

Objectives: To perform a systematic literature review on guidelines for MSUS for children published by international societies and articles on how to perform MSUS scanning in children. This represents the first step for an EULAR taskforce, which objective is to develop EULAR Standardized Procedures for UItrasound Imaging in Pediatric Rheumatology through a consensus process among rheumatologists, paediatric rheumatologists, and radiologists highly experienced in the performance, teaching and research in paediatric MSUS in rheumatologic disease.

Methods: The objective was reformulated according to the PICO-adapted approach, as follows: body parts, ultrasound, and scanning procedures. For each component several synonyms were used. Search limits were applied for animal studies and age. The literature search was performed in Medline and Embase from databases inception to 1st June 2016. References identified were imported into a bibliographic manager and duplicates were removed. To identify eligible studies the remaining articles were assessed by title and abstract. Only articles in English were retained. From the selected studies, data about the examined area, patient position, probe placement, scanning method, landmarks and pathologies using a predefined data collection form.

Results: The literature search resulted in 6059 articles, of which 4856 were captured in Medline and 1203 in Embase. Figure 1 shows the the study flow-chart for article selection. After removing duplicates and scanning titles and abstracts, 295 articles remained for detailed review. After full-text review, 107 articles were excluded. The main reason for article exclusion after full-text review was the lack of standardized examination. Of the remaining articles, 2 described shoulder structures, 14 elbow structures, 5 wrist structures, 9 hand structures, 25 hip

Figure 1. Flow-chart of the article selection process.

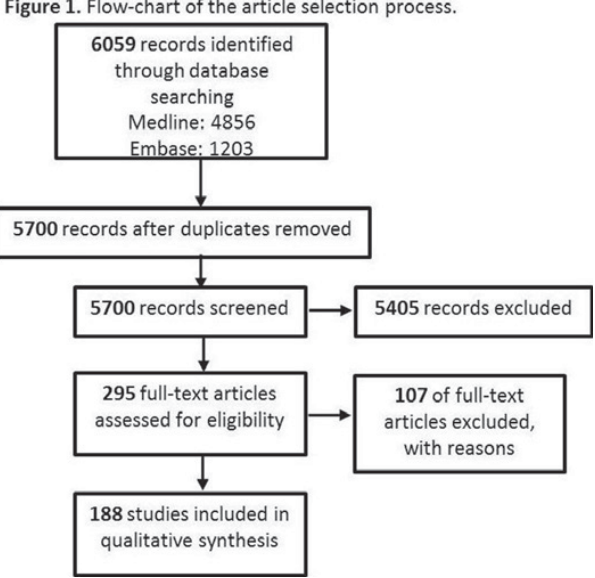

